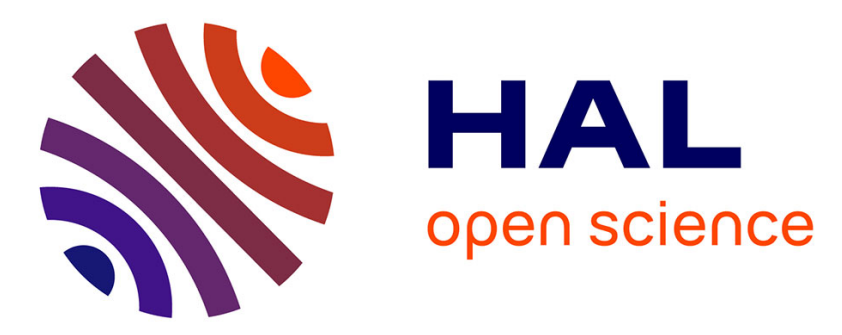

\title{
A Coupling Between the Facet Finite Element and Reluctance Network Methods in 3-D
}

Anderson Nunes, Olivier Chadebec, Patrick Kuo-Peng, Patrick Dular, Gérard Meunier

\section{- To cite this version:}

Anderson Nunes, Olivier Chadebec, Patrick Kuo-Peng, Patrick Dular, Gérard Meunier. A Coupling Between the Facet Finite Element and Reluctance Network Methods in 3-D. IEEE Transactions on Magnetics, 2017, 53 (10), pp.1 - 10. 10.1109/TMAG.2017.2723576 . hal-01692409

\section{HAL Id: hal-01692409 https://hal.science/hal-01692409}

Submitted on 4 Feb 2020

HAL is a multi-disciplinary open access archive for the deposit and dissemination of scientific research documents, whether they are published or not. The documents may come from teaching and research institutions in France or abroad, or from public or private research centers.
L'archive ouverte pluridisciplinaire HAL, est destinée au dépôt et à la diffusion de documents scientifiques de niveau recherche, publiés ou non, émanant des établissements d'enseignement et de recherche français ou étrangers, des laboratoires publics ou privés. 


\title{
A coupling between the Facet Finite Element and Reluctance Network Methods in 3-D
}

\author{
Anderson S. Nunes ${ }^{1,3,4}$, Olivier Chadebec ${ }^{2}$, Patrick Kuo-Peng ${ }^{3}$, Patrick Dular ${ }^{4}$, and Gérard Meunier ${ }^{2}$ \\ ${ }^{1}$ Engineering Simulation \& Scientific Software (ESSS), Rua Orlando Phillipi, $\mathrm{n}^{\circ} 100,1^{\circ}$ andar, \\ Florianópolis, CEP: 88032-700, Brazil \\ 2 Univ. Grenoble Alpes, CNRS, G2Elab, F-38000 Grenoble, France \\ ${ }^{3}$ GRUCAD/ UFSC - Universidade Federal de Santa Catarina, Florianópolis, CEP: 88040-970, Brasil \\ ${ }^{4}$ University of Liège, Department of Electrical Engineering and Computer Science, ACE, \\ Liège B-4000, Belgium
}

A 3-D Finite Element Method (FEM) mesh is converted to a reluctance network through an original magnetostatic formulation based on facet shape functions interpolation of the magnetic induction. This network is coupled with a standard reluctance network, characterizing a 0-D system and solved by a circuit solver approach.

Index Terms-Reluctance network, Finite Element Method (FEM), facet shape functions, Facet FEM (FFEM)

\section{INTRODUCTION}

$\mathbf{O}$ PTIMIZING electromagnetic devices can require a large amount of data which, if obtained by means of numerical analysis, can be a time-consuming task. Sometimes, only analyzing large size problems might be time-consuming, mainly when the models have high aspect ratio, for instance, the large electrical machines or actuators with small air gaps.

Many numerical methods [1] are available for a wide range of applications. The FEM (Finite Element Method) and the Reluctance Network Method (RNM) are the most widely used for magnetostatic modeling.

The FEM is well known for its flexibility and generality, since the mathematical formulations are solved based on a mesh. Furthermore, the literature available for this method is noticeable, for instance [2] [3]. However, it can lead to a high number of degrees of freedom and so quite long computation times [4].

On the other hand, the RNM is one of the most primitive methods for magnetic modeling and its application is based on a reluctance network. This method has remained useful due to its coherent results obtained with low computational effort and computational simulation time [5]. It has largely been applied to model power transformers [4], [6]-[8], rotating electrical machines [9]-[11] and transmission lines [12]. In general, the reluctance networks are defined manually, which is usually a hard, long and non-general task.

A methodology for coupling both methods would thus be of importance. Some portions of the studied domain could be described by lumped analytical reluctances, i.e., RNM, and the more complex regions could be automatically discretized and refined as necessary with FEM. This kind of method is known to offer a good trade-off between simulation accuracy and problem size, requiring less time if compared with the fully discretized method [13].

Manuscript received March 3, 2017. Accepted June 25, 2017. Corresponding author: A. Nunes (email: nunes.anderson@gmail.com).
In [14], a methodology that couples classical nodal/edge 3-D FEM with external reluctance networks is presented. It requires the definition of cuts along the flux tubes to manage the surface jumps of derived scalar potentials as well as the application of a gauge condition using the tree/co-tree technique. Furthermore, in [15], an approach is presented to couple reluctance networks with an FEM magnetic scalar potential formulation, which requires cuts to deal with multiply connected domains.

The conversion of an FEM mesh into an equivalent network has been discussed since [16]. It is presented using nodal, edge and facet elements in [16]-[22]. The FFEM (FEM with Whitney facet basis functions) is also applied in [23] and [24] to model static fields and seems to be an appropriate solution for the coupling with the RNM, since its application considering a magnetostatic formulation [24]-[26] generates an FFEM stiffness matrix that can be interpreted as a magnetic circuit [27] [28].

Considering all the complexities of applying the classical 3-D FEM formulations in complex domains and coupling these formulations with external reluctance networks, this paper aims to present an alternative solution. Once the FFEM matrix can be interpreted as a magnetic circuit and easily coupled with the RNM, this entire system, fully compatible with both numerical approaches, i.e., FEM and RNM, can be solved by a single 0 -D circuit solver that unifies the magnetic induction free divergence constraint. This network can be solved considering magnetomotive force $(\mathrm{mmf})$ sources from permanent magnets, coils or from external magnetic circuits. The flux sources are allowed in the external circuits and can also be used to impose magnetic induction fields along the boundaries of the discretized domain.

For this purpose, the FFEM formulation, the way to apply its boundary conditions (BCs) following the circuit formalism and the coupling of the network obtained through the FFEM with the external one are presented. The nodal and independent loop circuit approaches are analyzed and the FFEM formulation is 
compared with the classical edge A-formulation.

Finally, one simple case is used to illustrate the FFEM matrix and the coupling process. Then, the results of modeling an actuator with the classical FEM A-formulation and with the proposed methodology are compared.

\section{Magnetostatic Facet FEM Methodology}

\section{A. Basis equations}

The magnetostatic fields are described by the Ampere (1) and Gauss (2) equations, together with the magnetic constitutive relation for linear and isotropic materials (3), i.e.,

$$
\begin{gathered}
\operatorname{curl} \mathbf{H}=\mathbf{J}_{s} \quad(1) \quad \operatorname{div} \mathbf{B}=0 \\
\mathbf{B}=\mu \mathbf{H}+\mathbf{B}_{r}
\end{gathered}
$$

where $\mathbf{H}$ is the total magnetic field, $\mathbf{J}_{s}$ is the source current density, $\mathbf{B}$ is the magnetic induction, $\mu$ is the magnetic permeability and $\mathbf{B}_{r}$ is the remnant magnetic induction of permanent magnets.

Total field $\mathbf{H}$ can be split into two terms,

$$
\mathbf{H}=\mathbf{H}_{s}+\mathbf{H}_{r}
$$

where reaction field $\mathbf{H}_{r}$ is obtained from the reduced magnetic scalar potential $\psi$,

$$
\mathbf{H}_{r}=-\operatorname{grad} \psi
$$

and magnetic source field $\mathbf{H}_{s}$, satisfying

$$
\operatorname{curl} \mathbf{H}_{s}=\mathbf{J}_{s}
$$

can be obtained with the Biot-Savart equation, possibly adapted for particular geometries [29]-[32]. The source of field $\mathbf{H}_{s}$ can thus be outside the studied domain. If it is partially or completely inside the domain, conformal meshes are not required. Besides that, it is possible to use higher order elements for the integration process, increasing the accuracy of $\mathbf{H}_{s}$.

Applying (5) in (4), one has

$$
\mathbf{H}+\operatorname{grad} \psi=\mathbf{H}_{s}
$$

Applying (3) in (7), one obtains the strong formulation based on $\mathbf{B}$, i.e.,

$$
\frac{1}{\mu} \mathbf{B}+\operatorname{grad} \psi=\mathbf{H}_{s}+\frac{1}{\mu} \mathbf{B}_{r}
$$

\section{B. Facet shape functions}

The shape function spaces are defined using the Whitney forms [33] [25], where $W^{0}, W^{1}, W^{2}$ and $W^{3}$ are the nodal, edge, facet and volume function spaces, respectively. The relations between these spaces are given by the Rham's diagram [25],

$$
W^{0} \stackrel{\operatorname{grad} f}{\longrightarrow} W^{1} \stackrel{\text { curl } \mathbf{F}}{\longrightarrow} W^{2} \stackrel{\operatorname{div} \mathbf{F}}{\longrightarrow} W^{3}
$$

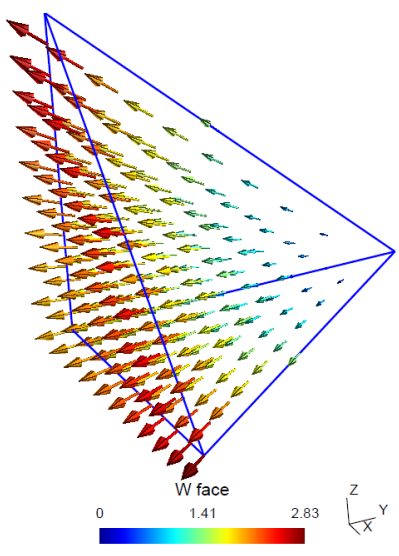

(a) Facet shape function

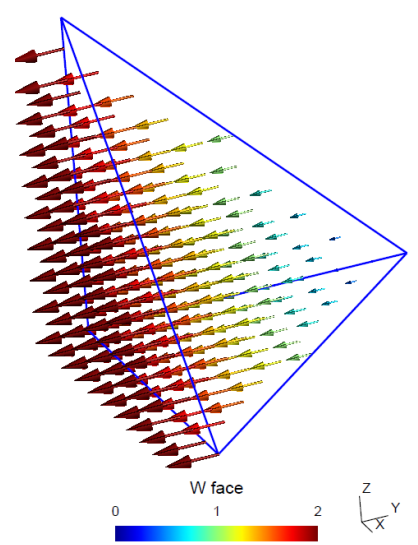

(b) Normal component
Fig. 1. Facet shape functions obtained for tetrahedral element

The facet shape function is plotted for the face parallel to the XZ-plane on a reference tetrahedral element, where the dimensions are unitary and the referred face has an area of 0.5 . Its behavior is depicted in Fig. 1a and its normal component is presented in Fig. 1b.

As illustrated in Fig. 1b, the flux is equal to 1 for a given face $i$

$$
\mathbf{W}_{i} \cdot \mathbf{n}_{i}=\frac{1}{S_{i}}
$$

and null along the other faces, Fig. 1a. $S_{i}$ is the surface area of face $i$.

Another important aspect of the facet function is that its divergence in an element is equal to the inverse of the volume $V_{e}$ of this element (for first order elements):

$$
\operatorname{div} \mathbf{W}_{i}=\frac{1}{V_{e}}
$$

\section{Magnetostatic formulation based on facet shape func-} tions

Projecting (8) on a domain $\Omega$ (Fig. 2) by the Galerkin method, with the facet shape functions $\mathbf{B}^{\prime} \in W^{2}$ as test functions, leads to (12)

$$
\begin{array}{r}
\int_{\Omega} \frac{1}{\mu} \mathbf{B} \cdot \mathbf{B}^{\prime} d \Omega+\int_{\Omega} \operatorname{grad} \psi \cdot \mathbf{B}^{\prime} d \Omega-\int_{\Omega} \mathbf{H}_{s} \cdot \mathbf{B}^{\prime} d \Omega- \\
\int_{\Omega} \frac{1}{\mu} \mathbf{B}_{r} \cdot \mathbf{B}^{\prime} d \Omega=0
\end{array}
$$

Since the magnetic induction $\mathbf{B}$ is given by

$$
\mathbf{B}=\sum_{i=1}^{n_{f}} \mathbf{B}_{i}^{\prime} \Phi_{i}
$$

for first order elements, the first term of the left side of (12) can be rewritten as:

$$
\int_{\Omega} \frac{1}{\mu} \mathbf{B} \cdot \mathbf{B}^{\prime} d \Omega=\sum_{j=1}^{n_{f}}\left(\int_{\Omega} \frac{1}{\mu} \mathbf{B}_{j} \cdot \mathbf{B}_{i}^{\prime} d \Omega\right) \Phi_{i}
$$


where $j$ and $i$ are the faces indices, $n_{f}$ is the number of faces and $\Phi_{i}$ is the magnetic flux through the face $i$. It is important to notice that (14) results in a self-reluctance for $i=j$ and in a mutual reluctance between faces $i$ and $j$ for $i \neq j$.

The second term of (12) is solved using the following vector identity

$$
\operatorname{div}(f \mathbf{A})=\operatorname{grad} f \cdot \mathbf{A}+f \operatorname{div} \mathbf{A}
$$

resulting in:

$$
\int_{\Omega} \operatorname{grad} \psi \cdot \mathbf{B}^{\prime} d \Omega=\int_{\Omega} \operatorname{div}\left(\psi \mathbf{B}^{\prime}\right) d \Omega-\int_{\Omega} \psi \operatorname{div} \mathbf{B}^{\prime} d \Omega
$$

Applying the divergence theorem in the second term of (16), one obtains

$$
\int_{\Omega} \operatorname{grad} \psi \cdot \mathbf{B}^{\prime} d \Omega=\int_{\Gamma} \psi \mathbf{B}^{\prime} \cdot \mathbf{n} d \Gamma-\int_{\Omega} \psi \operatorname{div} \mathbf{B}^{\prime} d \Omega
$$

where $\Gamma$ is the boundary of $\Omega$, Fig. 2 .

The surface integral term of (17) is null and needs to be analyzed in the following cases (See Fig. 2):

- External surface $\Gamma_{B}$ : imposes that field $\mathbf{B}$ only has tangential components on this surface;

- Internal faces of $\Omega$ : in accordance with the facet functions definitions, $\mathbf{B}^{\prime}$ is null everywhere except inside the two elements that share the face $i$;

- $\Gamma_{l} \cup \Gamma_{m}$ : This second case allows to limit $\Omega$ to the sum of $\Omega_{l}$ to $\Omega_{m}$, Fig. 2 and (18). However, $\mathbf{B}^{\prime} \cdot \mathbf{n}$ is null along $\Gamma_{l} \cup \Gamma_{m}$, except along $\Gamma_{l} \cap \Gamma_{m}$;

- $\Gamma_{l} \cap \Gamma_{m}$. The shape functions $\mathbf{B}_{l}^{\prime}$ and $\mathbf{B}_{m}^{\prime}$ have opposite directions along these faces, canceling each other out.

$$
\begin{gathered}
\int_{\Omega_{l}+\Omega_{m}} \operatorname{grad} \psi \cdot \mathbf{B}^{\prime} d \Omega=\int_{\Gamma_{l}+\Gamma_{m}} \psi \mathbf{B}^{\prime} \cdot \mathbf{n}_{\Gamma} d \Gamma- \\
\int_{\Omega_{l}+\Omega_{m}} \psi \operatorname{div} \mathbf{B}^{\prime} d \Omega
\end{gathered}
$$

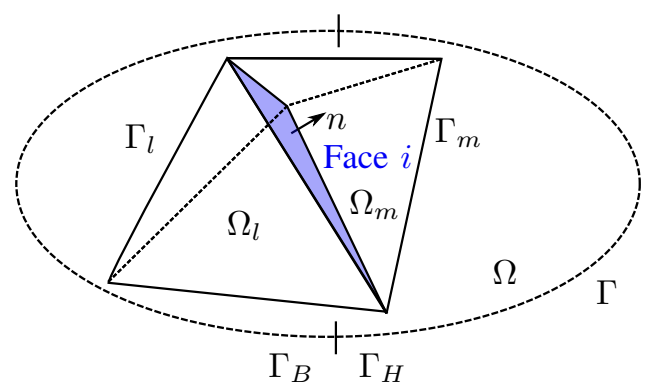

Fig. 2. The complete domain $\Omega$ with its outer surface $\Gamma=\Gamma_{H} \cup \Gamma_{B}$ and two adjacent elements $\Omega_{l}$ and $\Omega_{m}$, with their faces $\Gamma_{l}$ and $\Gamma_{m}$.

Thus, considering that inside each element $\Omega_{l}$ or $\Omega_{m}$, the reduced scalar potential $\psi_{l}$ or $\psi_{m}$ are constants since each of the elements represents one vertice in the dual mesh, i.e. one node in the reluctance network, and also defining the flux direction from $\Omega_{l}$ to $\Omega_{m}$, (18) becomes

$$
\begin{array}{r}
\int_{\Omega_{l}+\Omega_{m}} \operatorname{grad} \psi \cdot \mathbf{B}^{\prime} d \Omega= \\
\psi_{l} \int_{\Omega_{l}} \operatorname{div} \mathbf{B}^{\prime} d \Omega_{l}- \\
\psi_{m} \int_{\Omega_{m}} \operatorname{div} \mathbf{B}^{\prime} d \Omega_{m}
\end{array}
$$

The divergence of $\mathbf{B}^{\prime}$ being the inverse of the element volume, as given in (18), equation (19) becomes

$$
\int_{\Omega_{l}+\Omega_{m}} \operatorname{grad} \psi \cdot \mathbf{B}^{\prime} d \Omega=\psi_{l}-\psi_{m}
$$

Finally, (12) is rewritten as

$$
\begin{array}{r}
\sum_{j=1}^{n_{f}}\left(\int_{\Omega} \frac{1}{\mu} \mathbf{B}_{j} \cdot \mathbf{B}^{\prime}{ }_{i} d \Omega\right) \Phi_{i}+\psi_{a}-\psi_{b}= \\
\int_{\Omega} \mathbf{H}_{s} \cdot \mathbf{B}^{\prime} d \Omega+\int_{\Omega} \frac{1}{\mu} \mathbf{B}_{r} \cdot \mathbf{B}^{\prime} d \Omega
\end{array}
$$

or in a matrix form:

$$
[\Re][\Phi]-\left[\psi_{m}\right]=\left[\psi_{0}\right]
$$

where $[\Re]$ is the FFEM matrix, physically known as a reluctance matrix, $[\Phi]$ is the unknown flux matrix, $\left[\psi_{m}\right]$ is the magnetic potential between each reluctance and $\left[\psi_{0}\right]$ contains the $m m f$ branch sources, defined by means of $\mathbf{H}_{s}$ and $\mathbf{B}_{r}$. Another possibility to obtain this term would be to describe the conductors as stranded current loops, as presented in [18].

Even though the magnetic induction free divergence (2) has not been used to obtain the matrix system (22), it will be considered by solving this matrix system as a magnetic circuit, i.e., a 0-D system where its free divergence is imposed by the Kirchhoff's current law here applied to the magnetic flux.

\section{Implementation of $\left.\mathbf{n} \cdot \mathbf{B}\right|_{\Gamma_{B}}=0 B C s$}

The $\left.\mathbf{n} \cdot \mathbf{B}\right|_{\Gamma_{B}}=0$ BC, discussed in Section II-C, implies a zero magnetic flux through surface $\Gamma_{B}$. From the circuit standpoint, this means that the branches that represent the element surfaces on $\Gamma_{B}$ are open. So, they can be suppressed from the equations system, i.e., from the magnetic circuit. This process is exemplified in Fig. 6, where the gray reluctances are not taken into account.

\section{E. Implementation of $\mathbf{n} \times\left.\mathbf{H}\right|_{\Gamma_{H}}=0$ BCs}

The $\mathbf{n} \times\left.\mathbf{H}\right|_{\Gamma_{H}}=0$ BC can be used to apply symmetry planes and define the $\mathrm{BC}$ along coupling surfaces.

This is achieved by creating one external node $P$ ( Fig. 3) and connecting it with all the elements along $\Gamma_{H}$, which consists in imposing a constant magnetic scalar potential on this boundary, (23).

$$
\mathbf{n} \times\left.\mathbf{H}\right|_{\Gamma_{H}}=-\mathbf{n} \times\left.\operatorname{grad} \psi\right|_{\Gamma_{H}}=0
$$




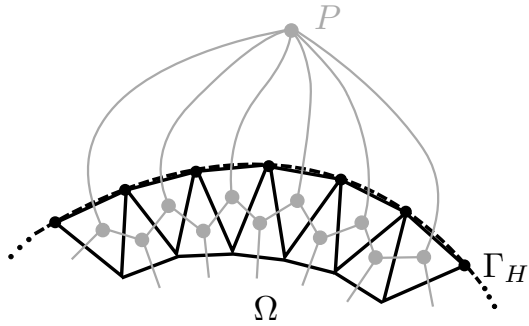

Fig. 3. $\mathrm{BC} \mathbf{n} \times\left.\mathbf{H}\right|_{\Gamma_{\mathbf{H}}}=0$

If the node $P$ is not connected to any other external branch, as presented in Fig. 3, it leads to the magnetic induction free divergence along this surface.

\section{F. Coupling with the external network}

The node $P$ (Fig. 3) can be used to connect the meshed domain $\Omega$ (converted into a reluctance network) to the external magnetic circuit, as depicted in Fig. 4, where $\Gamma_{c}$ is the connection surface. As $\Gamma_{c}$ is defined in the same manner as $\Gamma_{H}$ (Fig. 3 and (23)), the $\mathbf{n} \times\left.\mathbf{H}\right|_{\Gamma_{H}}=0 \mathrm{BC}$ is also applied on $\Gamma_{c}$.

In terms of implementation, this coupling is performed by adding the external network branches/nodes into the incidence matrix that represents the FFEM system, as well as by adding the external sources and reluctances into their respective matrices presented in (22).

This appears as an easy process, due to the circuit formalism. Thus, with both networks coupled, i.e., $\Omega+$ external network, the entire model can be solved as a single 0-D system.

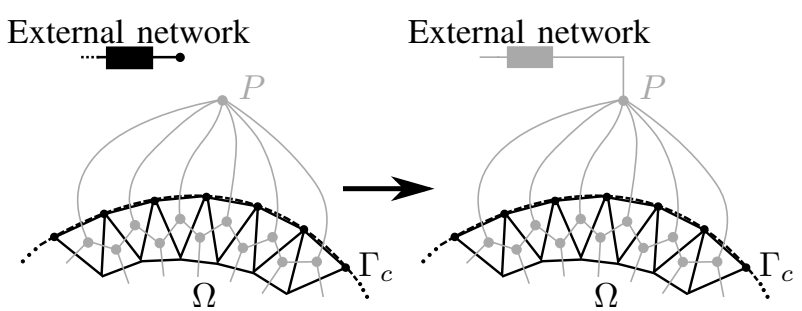

Fig. 4. Connection between the networks

\section{G. Circuit modeling}

The resulting circuit can be solved using both independent loop and nodal approaches [34]. Given a tetrahedral mesh with $n$ elements and $e_{f}$ external surfaces, the circuit characteristics can be analyzed through

$$
b=2 n+\frac{e_{f}}{2}
$$

$$
b=i+n-1
$$

where $b,(24)$, is number of branches in the dual mesh, i.e., faces in the mesh [35]. Thus, thanks to (24) and (25), the number of independent loops $i$ can be obtained,

$$
i=n+\frac{e_{f}}{2}+1
$$

So, we may remark that, depending on the $\mathrm{BCs}$, the independent loop approach can generate only a few more unknowns, $i$, than the nodal approach, which generates $n$ unknowns. Nonetheless, the independent loop approach requires an algorithm capable of defining the tree/co-tree, for instance, the Welsh algorithm [2] or the method proposed in [36]. As mentioned in [26], this process can be timeconsuming in larger meshes. Whereas, the nodal approach does not require any other algorithm to find the tree/co-tree, but it requires the inversion of the reluctance matrix, which can be computationally heavy. Furthermore, since this inversion generates a full matrix, it would impact the performance of sparse matrix tools possibly applied.

After evaluating the solving time, the nodal approach has been used to solve the magnetic circuits, thereby corroborating with the conclusion presented in [16]. In the future, this analysis will be extended to nonlinear materials, where the circuit approach impacts on the number of iterations of the nonlinear solvers [37].

\section{H. Comparison with A-formulation}

The A-formulation based on edge elements is very common in magnetostatic modeling and its comparison with the $\mathbf{B}$ formulation used in this work is of importance. For this purpose, the A weak formulation, with test function $\mathbf{A}^{\prime} \in W^{1}$, is

$$
\begin{array}{r}
\int_{\Omega} \frac{1}{\mu} \operatorname{curl} \mathbf{A} \cdot \operatorname{curl} \mathbf{A}^{\prime} d \Omega-\int_{\Omega} \frac{1}{\mu} \mathbf{B r} \cdot \operatorname{curl} \mathbf{A}^{\prime} d \Omega+ \\
\int_{\Gamma}(\mathbf{n} \times \mathbf{H}) \cdot \mathbf{A}^{\prime} d \Gamma=\int_{\Omega} \mathbf{J} \cdot \mathbf{A}^{\prime} d \Omega
\end{array}
$$

Through the functional spaces relation curl $\mathbf{A}^{\prime}=\mathbf{B}^{\prime}$ and (1), (27) can be rewritten as:

$$
\begin{aligned}
& \int_{\Omega} \frac{1}{\mu} \operatorname{curl} \mathbf{A} \cdot \mathbf{B}^{\prime} d \Omega-\int_{\Omega} \frac{1}{\mu} \mathbf{B r} \cdot \mathbf{B}^{\prime} d \Omega+ \\
& \int_{\Gamma}(\mathbf{n} \times \mathbf{H}) \cdot \mathbf{A}^{\prime} d \Gamma=\int_{\Omega} \operatorname{curl} \mathbf{H} \cdot \mathbf{A}^{\prime} d \Omega
\end{aligned}
$$

Expressing $\mathbf{A}$ in terms of $\mathbf{B}(\mathbf{B}=\operatorname{curl} \mathbf{A})$, leads to

$$
\begin{array}{r}
\int_{\Omega} \frac{1}{\mu} \mathbf{B} \cdot \mathbf{B}^{\prime} d \Omega-\int_{\Omega} \frac{1}{\mu} \mathbf{B r} \cdot \mathbf{B}^{\prime} d \Omega+ \\
\int_{\Gamma}(\mathbf{n} \times \mathbf{H}) \cdot \mathbf{A}^{\prime} d \Gamma=\int_{\Omega} \operatorname{curl} \mathbf{H} \cdot \mathbf{A}^{\prime} d \Omega
\end{array}
$$

In the surface integral term, one may rewrite $(\mathbf{n} \times \mathbf{H}) \cdot \mathbf{A}^{\prime}$ as $-\left(\mathbf{n} \times \mathbf{A}^{\prime}\right) \cdot \mathbf{H}$ that is null because of the BC $\mathbf{n} \times\left.\mathbf{A}^{\prime}\right|_{\Gamma_{B}}=0$. So, considering the boundaries conditions for the magnetic induction $\mathbf{B}$, the formulations are equivalent, since $\mathbf{n} \times\left.\mathbf{A}^{\prime}\right|_{\Gamma_{B}}=$ $\left.\mathbf{n} \cdot \mathbf{B}^{\prime}\right|_{\Gamma_{B}}=0$.

Regarding the last term of (29), it is possible to apply the Green equation of type curl $-\operatorname{curl}$ and $\operatorname{curl} \mathbf{A}^{\prime}=\mathbf{B}^{\prime}$, 


$$
\int_{\Omega} \operatorname{curl} \mathbf{H} \cdot \mathbf{A}^{\prime} d \Omega=\int_{\Omega} \mathbf{H} \cdot \mathbf{B}^{\prime} d \Omega+\int_{\Gamma}\left(\mathbf{A}^{\prime} \times \mathbf{n}\right) \cdot \mathbf{H} d \Omega
$$

where the last term is null and the source field $\mathbf{H}_{s}$ is obtained with the Biot-Savart equation, $\operatorname{curl} \mathbf{H}_{r}=0$,

$$
\int_{\Omega} \operatorname{curl} \mathbf{H} \cdot \mathbf{A}^{\prime} d \Omega=\int_{\Omega} \mathbf{H}_{s} \cdot \mathbf{B}^{\prime} d \Omega
$$

Both formulations appear to be equivalent, but considering that they are implemented in different ways, it is worthwhile analyzing others aspects: a) considering the number of edges in a regular tetrahedral mesh [35] and the number of unknowns per edge of the A-formulation [38], it was found out that the total number of unknowns are similar in both formulations, b) A-formulation requires tree gauge conditions while $\mathbf{B}$ formulation requires the incidence matrix.

\section{MODELS AND RESULTS}

The models used to verify the proposed methodology follow an order of complexity. The first one is related to a simple 2$\mathrm{D}$ triangular mesh of an air gap without leakage flux. Then a 3-D trivial model is used to compare the convergence of both formulations. The last two are 3-D magnetic devices, where the magnetic core is simplified using the RNM, i.e. it is modeled by a simple magnetic circuit manually defined, and only the air gap regions are meshed and solved with FFEM. The results are compared with those obtained through FFEM considering the complete model.

The geometries and meshes are generated with the Gmsh program [39] and the $\mathbf{A}$-formulation is solved using the GetDP [40] code.

\section{A. Air gap without leakage flux}

As presented in Fig. 5, the 2-D air gap model is defined by one region with the same dimensions and without leakage flux. This is coupled with an external network, where the reluctances are neglected $(\Re=0)$. For the sake of simplicity, the relative magnetic permeability is considered unitary and the $m m f$ source is arbitrarily defined as $2500 \mathrm{~A}$, resulting in a magnetic flux of $2500 \mathrm{~Wb}$.

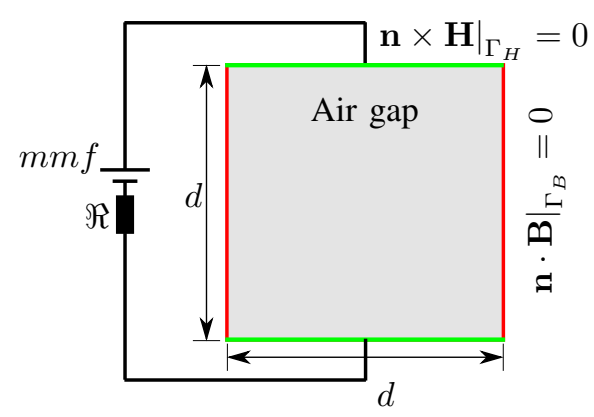

Fig. 5. Connection between the networks
The internal network obtained using FFEM is shown in Fig. 6. This network is then coupled to an external one, resulting in a single circuit.

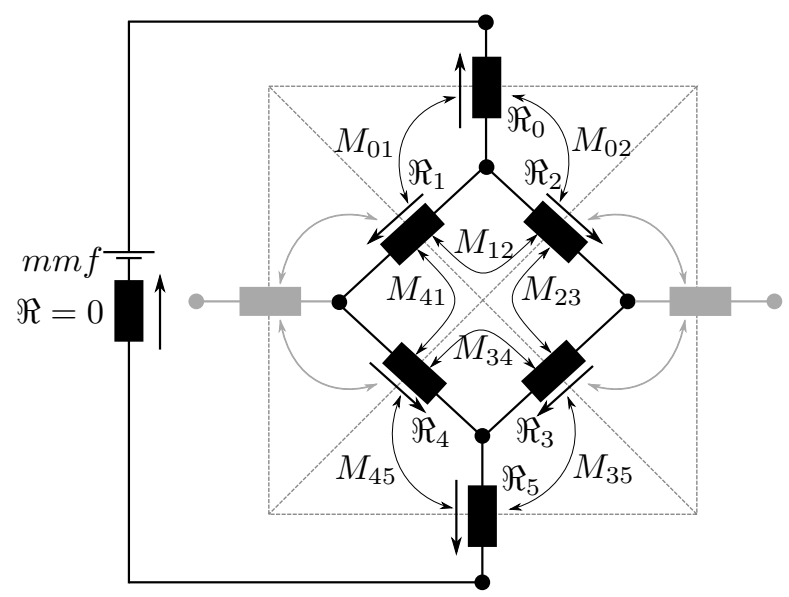

Fig. 6. Meshed domain coupled with the external network

The magnetic circuit is solved and the fluxes values are presented in Table I. The flux values and directions are in accordance with the circuit presented in Fig. 6.

TABLE I

Magnetic FluXes

\begin{tabular}{cc}
\hline \hline Reluctance index & $\begin{array}{c}\text { Magnetic flux [Wb] } \\
\times 10^{3}\end{array}$ \\
\hline 0 & -2.50 \\
1 & 1.25 \\
2 & 1.25 \\
3 & 1.25 \\
4 & 1.25 \\
5 & 2.50 \\
\hline \hline
\end{tabular}

\section{B. Convergence comparison}

As presented in Section II-H, the B and A-formulations are similar, consequently presenting the same level of convergence. For the sake of comparison, a simple 3-D model composed by two cubes is created and the dimensions are given in Fig. 7. The inner cube is a PM (permanent magnet) with a coercive field of $890000 \mathrm{~A} / \mathrm{m}$ and relative permeability of 1.09978 , while the outer one is a surrounding air domain.

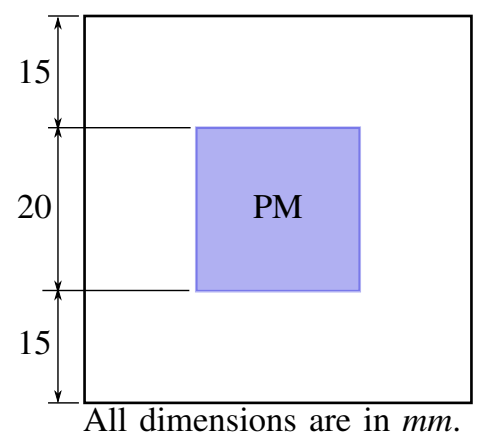

Fig. 7. Geometry for the convergence test 
The convergence is analyzed considering the magnetic energy calculated for a range of different meshes, from 96 up to 14482 elements, and comparing these values with the energy calculated with a very refined mesh, with 524175 elements. The results are presented in Fig. 8.

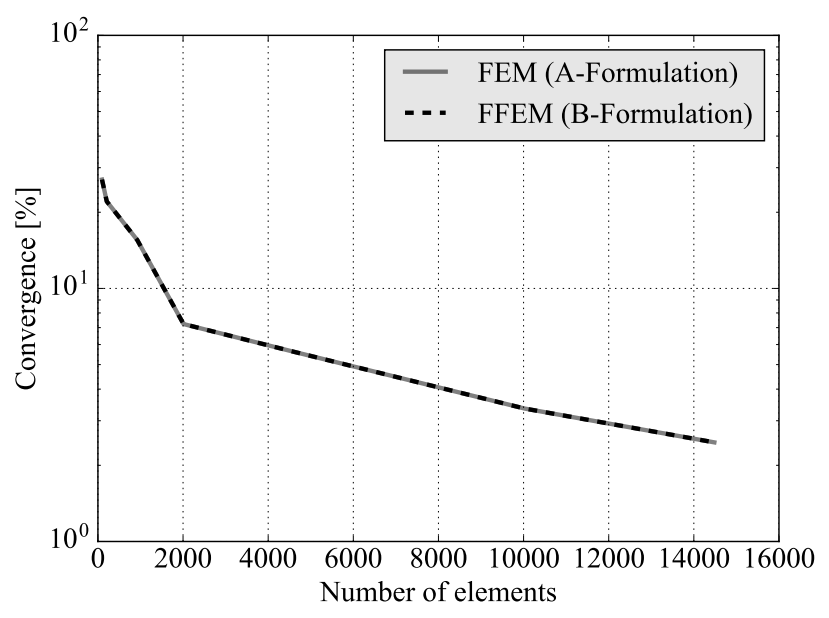

Fig. 8. Convergence comparison

\section{Magnetic device}

A more realistic 3-D magnetic device is now considered. It is composed of one winding and two magnetic regions separated by an air gap (Fig. 9). The magnetic permeability of the core is $\mu=2000 \mu_{0}$ and the winding is excited with $N I=3000 A$. The depth of the magnetic parts, air gap regions, and the outer air domain are 20,50 and $60 \mathrm{~mm}$, respectively. One probe line is placed at mid-thickness of the air gap region.

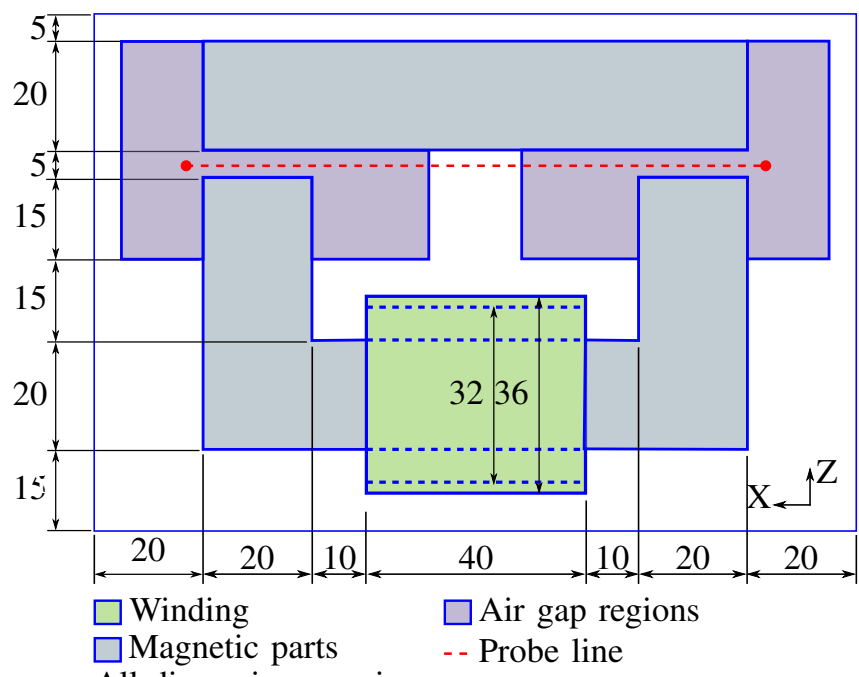

Fig. 9. Geometry - horizontal winding

As an additional convergence test, the problem is solved considering a coarse and a fine meshes made of 4151 and 14928 tetrahedral elements respectively. For the fine mesh, the magnetic inductions calculated with both formulations are shown in Fig. 10 and Fig. 11. For the coarse and fine meshes, their Z-components are plotted along the probe line, showing a good matching (Fig. 12). B and A-formulations generate practically the same number of unknowns, 14928 and 14921, respectively, for the fine mesh.

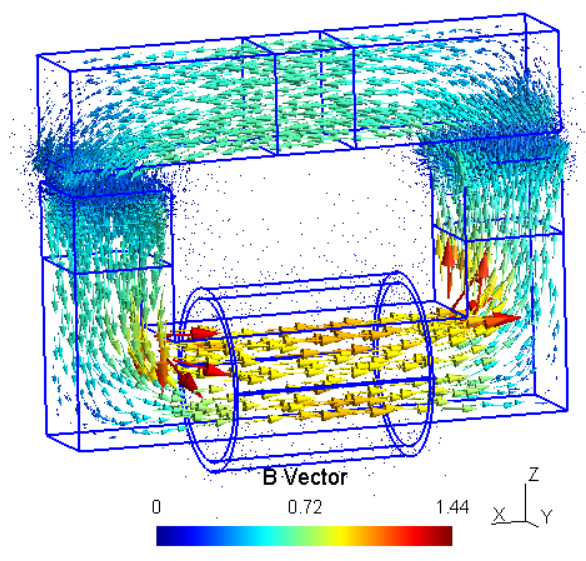

Fig. 10. Magnetic induction distribution - FFEM (B-formulation)

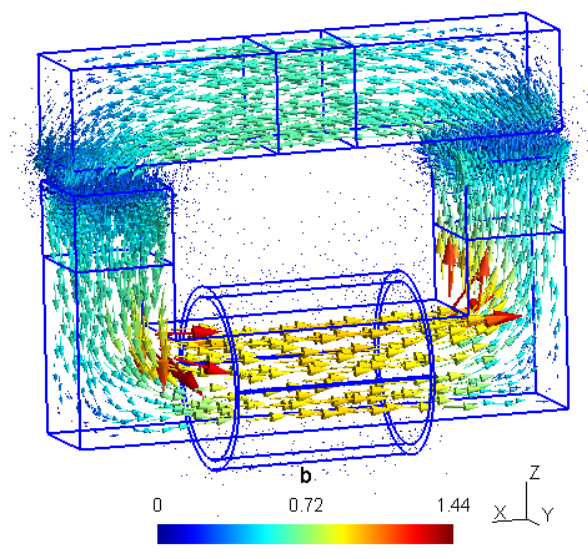

Fig. 11. Magnetic induction distribution - FEM (A-formulation)

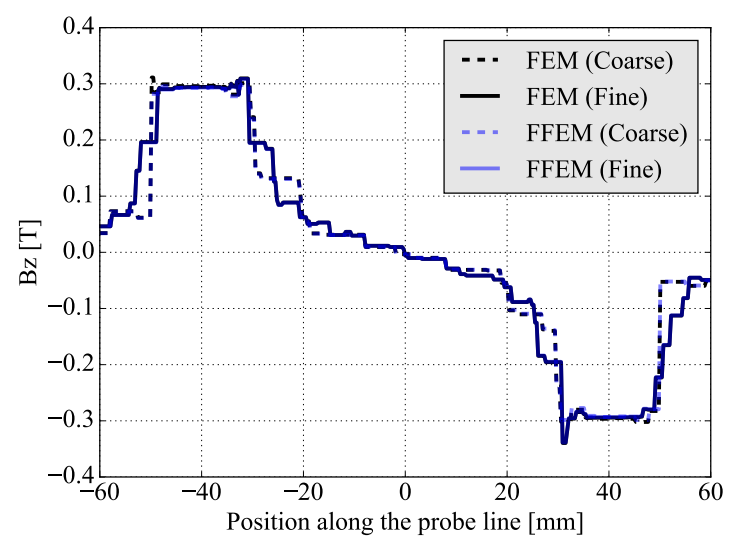

Fig. 12. $B_{z}$ along the probe line

The magnetic circuit is then simplified as a coupling of a classical reluctance network and the air gap regions (defined in 


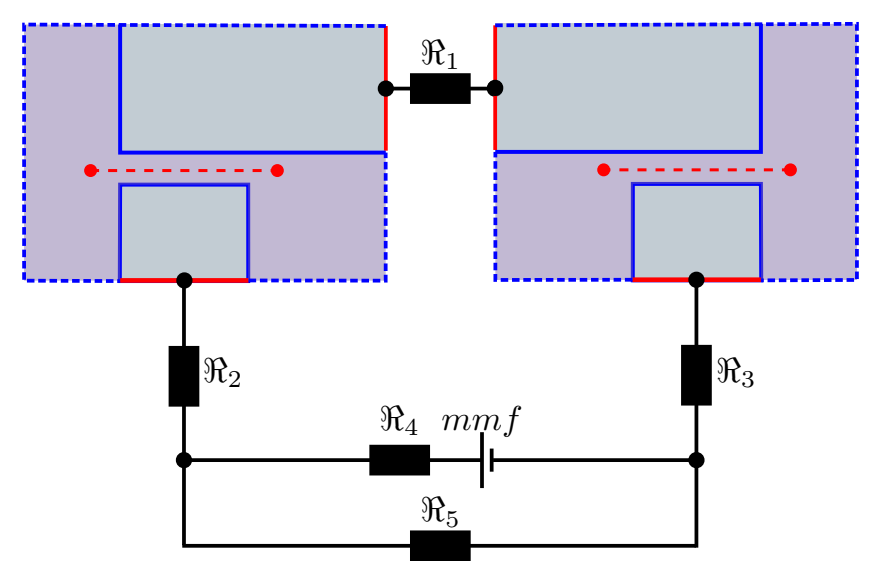

(a) Coupling 1

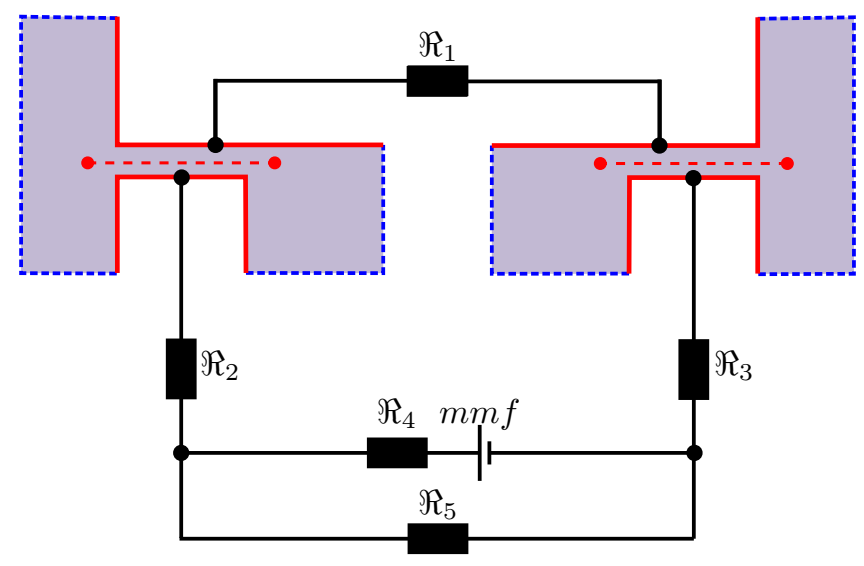

(b) Coupling 1

Fig. 13. Coupling between FFEM and RNM

Fig. 9), the latter asking for a more accurate model, here with FFEM, due to the leakage flux. Two cases with different levels of simplifications are considered (Fig. 13), taking account of some core portions or not:

- coupling 1 (Fig. 13a): some core portions are kept in the FFEM model and their connection surfaces with the RNM model are the core cross-sections intersected by the boundary of the reduced FFEM domain;

- coupling 2 (Fig. 13b): the core portions are removed from the FFEM model and their connection surfaces are the core outer surfaces used in coupling 1; only the air regions are discretized.

In Fig. 13, the connection surfaces $\Gamma_{c}$ are represented by continuous red lines and the surfaces with $\left.\mathbf{n} \cdot \mathbf{B}\right|_{\Gamma_{B}}=0 \mathrm{BC}$ are represented by the dashed blue lines. The reluctances $\Re_{1 \ldots 4}$ are for the core portions not considered in the FFEM domains and $\Re_{5}$ approximates the winding leakage flux path.

The meshes used for these models (Fig. 14) are clones of the fine mesh of the related portions in the complete model.

Both coupled problems are solved and compared in terms of the relative computational effort and accuracy. The number of unknowns, solution time and the magnetic flux through the upper magnetic part are compared in Tables II and III. In the complete model, the flux is obtained integrating the magnetic induction along the cross section of the upper magnetic part, while the flux through $\Re_{1}$ is considered for the problems

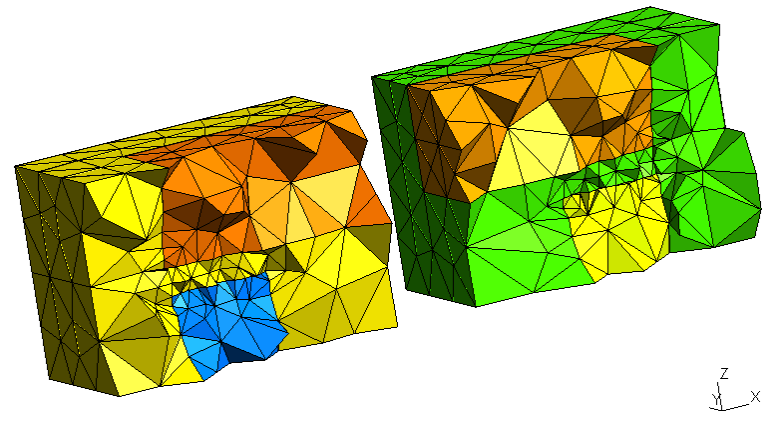

(a) Coupling 1

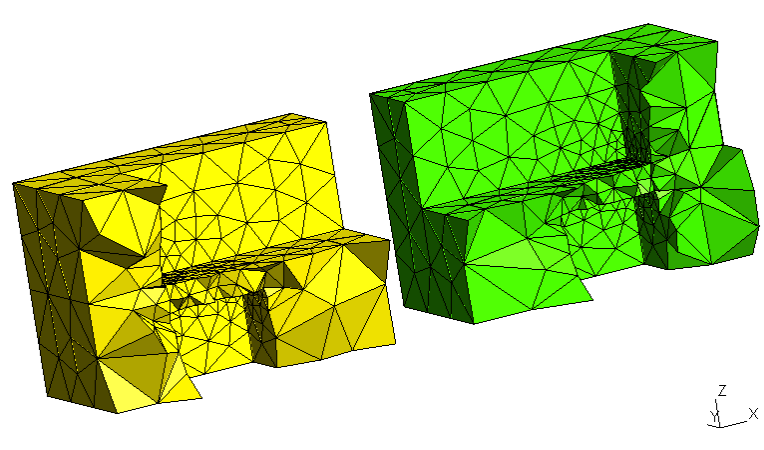

(b) Coupling 2

Fig. 14. Meshes of the air gap regions (with clipping)

coupling 1 and 2.

TABLE II

COMPUTATIONAL EFFORTS OF RNM-FFEM COUPLED MODELS COMPARED TO THE FULL FFEM MODEL

\begin{tabular}{cccc}
\hline \hline Method & $\begin{array}{c}\text { Number of } \\
\text { unknowns }\end{array}$ & $\begin{array}{c}\text { Number of } \\
\text { unknowns [\%] }\end{array}$ & $\begin{array}{c}\text { Solution } \\
\text { time [\%] }\end{array}$ \\
\hline FFEM (Fine) & 14928 & Ref. & $\begin{array}{c}\text { Ref. } \\
\text { Coupling 1 }\end{array}$ \\
Coupling 2 & 5257 & 55.31 & 17.09 \\
\hline \hline
\end{tabular}

TABLE III

ACCURACY OF RNM-FFEM COUPLED MODELS COMPARED TO THE FULL FFEM MODEL

\begin{tabular}{ccc}
\hline \hline Method & $\begin{array}{c}\text { Magnetic flux [Wb] } \\
\times 10^{-4}\end{array}$ & Dif. [\%] \\
\hline FFEM (Fine) & 2.5310 & Ref. \\
Coupling 1 & 2.520 & 0.553 \\
Coupling 2 & 2.515 & 0.632 \\
\hline \hline
\end{tabular}

As a local comparison, the Z-component of $\mathbf{B}$ along the probe lines (Fig. 13) is shown in Fig. 15 for all models. The relative differences of this solution between the coupled and full models are shown in Fig. 16. 


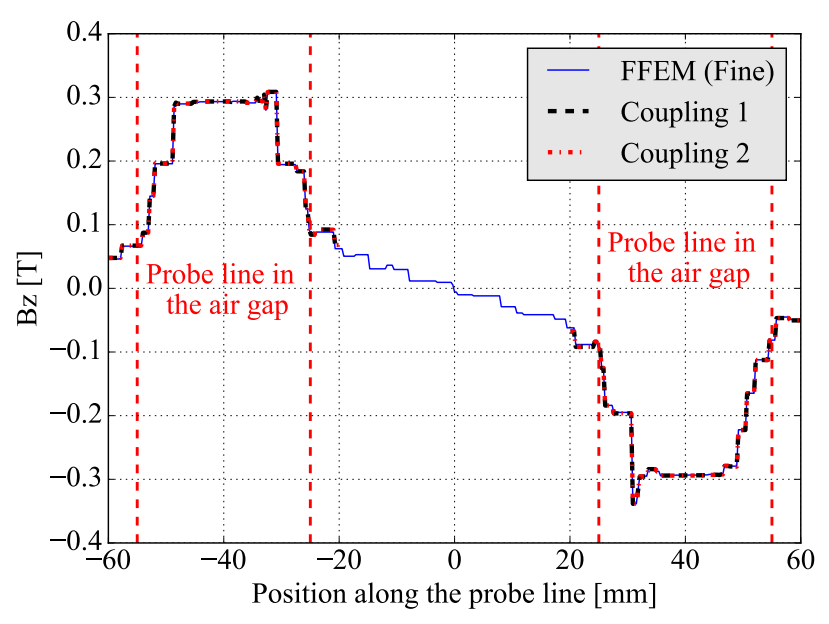

Fig. 15. $B_{z}$ obtained with the FFEM+RNM coupling

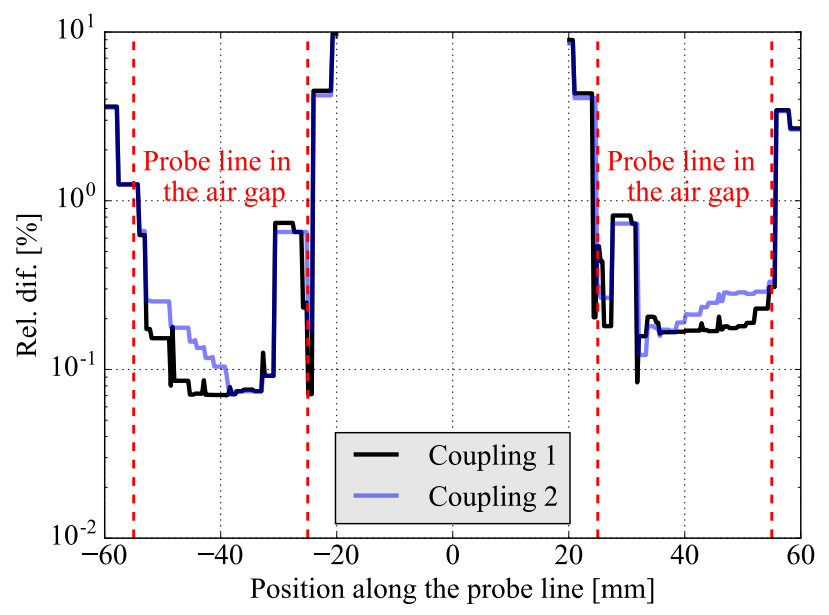

Fig. 16. Relative comparison of $B_{z}$

The differences are small in the region of interest (around $0.1 \%)$. However, they increase when the analysis point goes towards the $\left.\mathbf{n} \cdot \mathbf{B}\right|_{\Gamma_{B}}=0 \mathrm{BC}$, which is a natural consequence since the leakage flux is not totally in the Z-direction. Even though a weak field is computed close to those regions, this behavior cannot be desirable and can be minimized by increasing the $\mathrm{X}$ dimensions of the air regions, possibly unifying the domains. A more interesting and definitive solution would be to apply the "infinite" finite elements theory [41] in the X direction on those surfaces.

As expected, the 'coupling 2' problem presents higher differences, but the results are still coherent and acceptable.

As the solution along the meshed domains is obtained with the FFEM, it is possible to post-process the fields. So, the magnetic induction distribution is obtained in those domains and a zoom in the air gap is presented in Fig. 17. Once the plots are in the same color scale, it is possible to see that the Coupling 2 problem is properly modeling the leakage flux in the air gap.

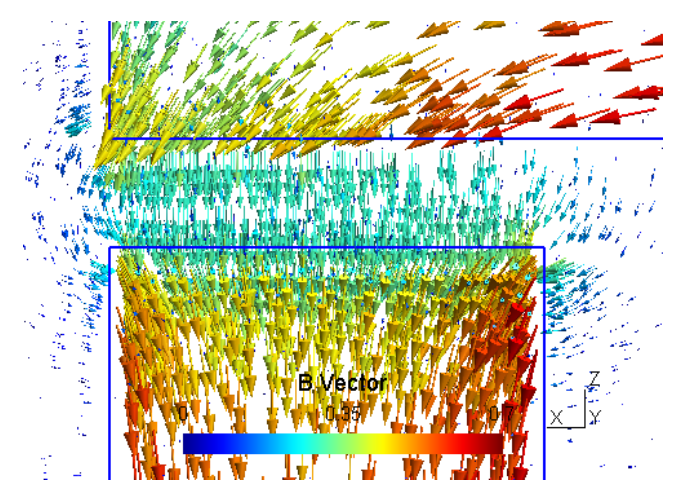

(a) Coupling 1

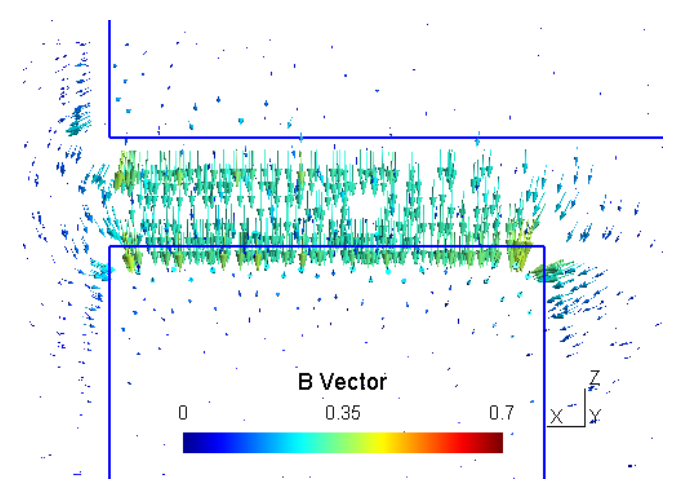

(b) Coupling 2

Fig. 17. Magnetic induction distribution along the discretized domains - Zoom in the air gap

In these coupled models, the winding is completely outside the meshed domain. However, it is possible to consider the windings inside or even partially inside of it. This last possibility is achievable because the SF is calculated independently, in this case using Biot-Savart, and thanks to the circuit coupling. It allows reducing the studied domain even when it does not have symmetry, needing only the connection surfaces. This is an important advantage over the classical formulations.

In order to illustrate this possibility, the model presented in Fig. 9 is modified as shown Fig. 18, where the windings are arranged vertically. The dimensions are the same as the model shown in Fig. 9, except the height of the windings which is $22 \mathrm{~mm}$.

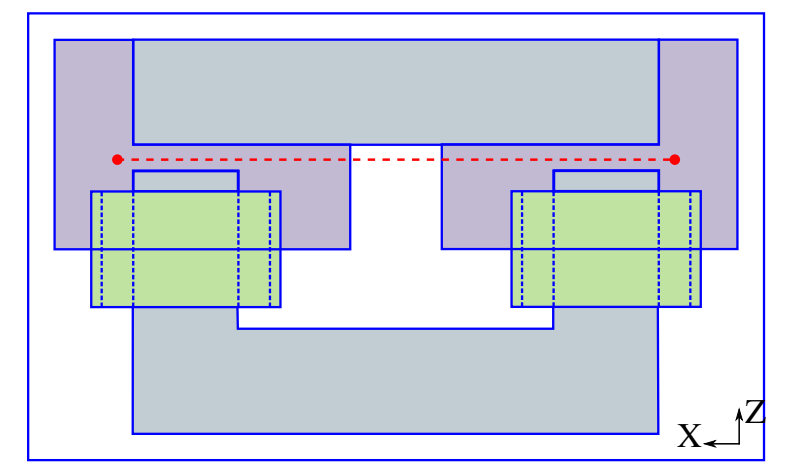

Fig. 18. Geometry - Vertical windings

Because the source field cannot be neglected on the connection surfaces $\Gamma_{c}$ used in the cut of the upper magnetic part (Fig. 
17a), an $m m f$ distribution can appear in the elements adjacent to these surfaces. Consequently, $\left.\operatorname{grad} \psi\right|_{\Gamma_{H}} \neq 0$, which does not respect the assumption defined in Section II-D.

This model is thus simplified as shown in Fig. 19, where the half of the windings are inside the meshed domains and the others halves of the Ampere-turns are considered as $m m f$ sources in the external circuit.

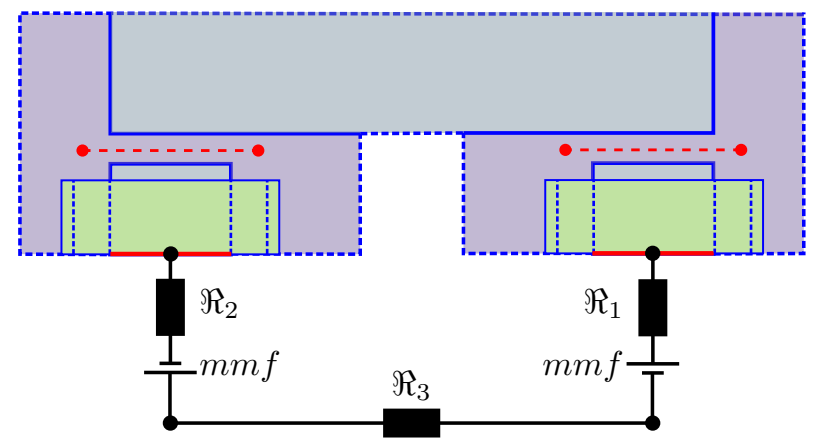

Fig. 19. Coupling FFEM+RNM

The source field is obtained along the entire domain and considering both parts of the windings. This problem is solved with the proposed coupling methodology and the magnetic induction distribution is presented in Fig. 20. Then, it is compared with the results obtained with the FEM A-formulation solved in the complete model, showing a good correlation in the regions of interest (Fig. 21).

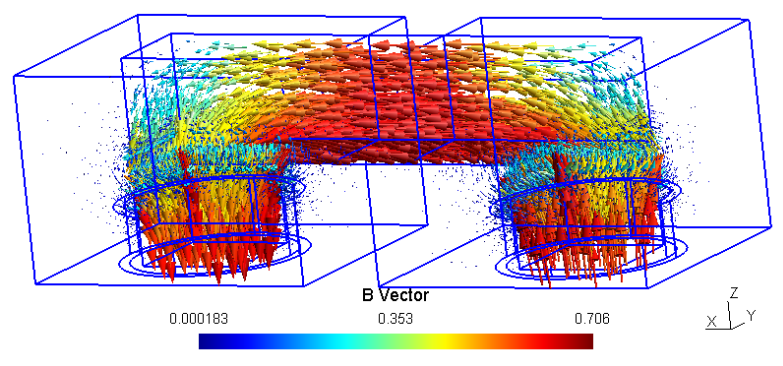

Fig. 20. Magnetic induction distribution

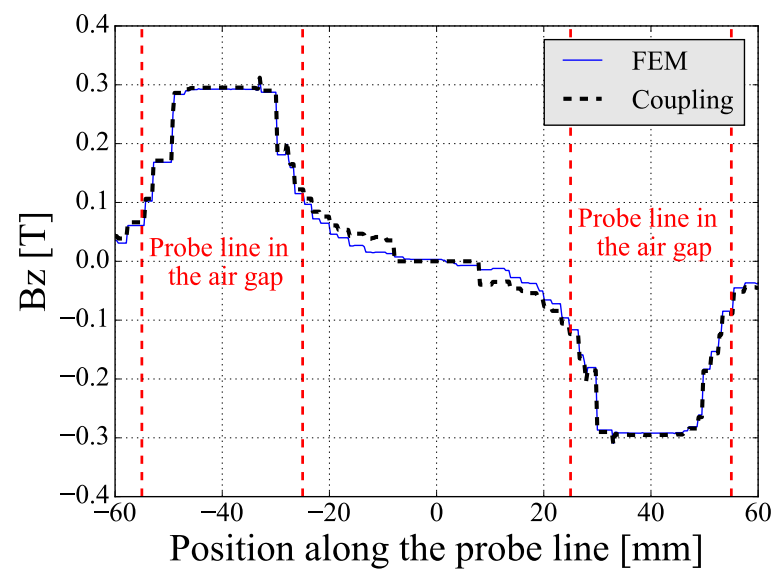

Fig. 21. $B_{z}$ along the probe line
The number of unknowns was reduced from 19982 (FEM A-formulation) to 13717 (FFEM+RNM).

\section{CONCLUSION}

A methodology capable of converting an FEM mesh into a reluctance network, allowing its easy coupling with the RNM, has been presented. As the circuit works as a gauge condition and the formulations are equivalent, it has been possible to keep the accuracy obtained with the $\mathbf{A}$-formulation, but without worrying about the cuts needed to deal with multiply connected domains.

The FFEM analyzed regions have been discretized with a mesh fine enough to ensure the accuracy level and the other outer regions have been simplified using the RNM. Even though only one type of flux tube was used in this work, it allows to use all the range of flux tubes available for this method [42]-[45].

Even if the representation of an FEM mesh in terms of a reluctance network has already been shown before, [16] for instance, a didactic case (Fig. 6) has been presented in order to show the coupling of this network with an external one, contributing to a different and simpler way to understand the numerical analysis. Additionally, two real cases with different windings arrangements, i.e. horizontal and vertical, were studied. In the last one, only part of the windings was considered inside the studied domain. Such capability allows a wide range of new possibilities to apply this coupling.

With the proposed methodology, the modeling domain can be drastically reduced, meshing only the regions with complex magnetic flux behavior. Two levels of simplifications have been presented, reducing the number of unknowns to $35 \%$ and the solving time to $7 \%$ in comparison with the complete model, keeping the quality of the results along the region of interest.

This methodology seems to provide more flexibility when dealing with the trade-off simulation time vs. accuracy, mainly for optimizations processes and even when analyzing large models.

It can easily be applied to other static physics with electric circuit analogy, like heat and current conduction [23].

\section{ACKNOWLEDGMENT}

This work is supported by CNPq (Conselho Nacional de Desenvolvimento Científico e Tecnológico - Brazil), project 200215/2016-7.

Anderson Nunes acknowledges Engineering Simulation and Scientific Software Ltda. (ESSS) for the help during this work.

\section{REFERENCES}

[1] E. Tonti, "Why starting from differential equations for computational physics?" Journal of Computational Physics, vol. 257, pp. 1260-1290, 2014.

[2] J. P. A. Bastos and N. Sadowski, Electromagnetic Modeling by Finite Element Methods. New York: Marcel Dekker, 2003.

[3] _ - Magnetic Materials and 3D Finite Element Modeling. Boca Raton: CRC Press, 2014.

[4] J. Turowski, "Rapid calculation of stray fields and losses in power transformers," ELEKTRYKA, p. 18, 2005. 
[5] J. Sykulski, Ed., Computational magnetics, 1st ed. Singapore: Springer, 1995, ch. Reluctance Networks, pp. 145-169.

[6] J. Turowski, I. Kraj, and K. Kulasek, "Industrial verification of rapid design methods in power transformers," Proc. TRANSFORMER, pp. 56, 2001

[7] A. Soto, D. Souto, J. Turowski, X. M. Lopez-Fernandez, and D. Couto, "Software for fast interactive three-dimensional modeling of electromagnetic leakage field and magnetic shunts design in Shell type transformers," in 2008 18th International Conference on Electrical Machines. IEEE, Sep. 2008, pp. 1-6.

[8] X. M. Lopez-Fernandez, P. Penabad-Duran, and J. Turowski, "3-D methodology for the heating hazard assessment on transformer covers," Sep. 2010.

[9] D. Petrichenko, "Contribution à la modélisation et à la conception optimale des turbo-alternateurs de faible puissance," phdthesis, École Centrale de Lille, 2007.

[10] M. Fukuoka, K. Nakamura, and O. Ichinokura, "A method for optimizing the design of SPM type magnetic gear based on reluctance network analysis," in 2012 XXth International Conference on Electrical Machines. IEEE, Sep. 2012, pp. 30-35.

[11] Y. Tang, T. E. Motoasca, J. J. H. Paulides, and E. A. Lomonova, "Analytical modeling of flux-switching machines using variable global reluctance networks," 2012 XXth International Conference on Electrical Machines, Sep. 2012.

[12] D. Bormann and H. Tavakoli, "Reluctance Network Treatment of Skin and Proximity Effects in Multi-Conductor Transmission Lines," IEEE Transactions on Magnetics, vol. 48, no. 2, pp. 735-738, Feb. 2012

[13] H. D. Gersem, K. Hameyer, and T. Weiland, "Field - circuit coupled models in electromagnetic simulation," Journal of Computational and Applied Mathematics, vol. 168, no. 1-2, pp. 125-133, Jul. 2004.

[14] P. Dular, J. Gyselinck, T. Henneron, and F. Piriou, "Dual finite element formulations for lumped reluctances coupling," IEEE Transactions on Magnetics, vol. 41, no. 5, pp. 1396-1399, May 2005.

[15] A. Lehikoinen and A. Arkkio, "A Systematic Approach for Solving Coupled Reluctance Network and Finite Element Models," in 20h Compumag Conference on the Computation of Electromagnetic Fields, no. 3, 2015, pp. 76-77.

[16] A. Demenko, L. Nowak, and W. Szelag, "Reluctance network formed by means of edge element method," IEEE Transactions on Magnetics, vol. 34 , no. 5, pp. 2485-2488, 1998.

[17] A. Demenko and J. Sykulski, "Network equivalents of nodal and edge elements in electromagnetics," IEEE Transactions on Magnetics, vol. 38, no. 2, pp. 1305-1308, Mar. 2002.

[18] A. Demenko, "Representation of windings in the $3 \mathrm{~d}$ finite element description of electromagnetic converters," in Proceedings of CEM 2002 - 4th International Conference on Computation in Electromagnetics. IEE, 2002

[19] _ "Network models of three-dimensional electromagnetic fields," in IET 7th International Conference on Computation in Electromagnetics (CEM 2008). IEE, 2008, pp. 70-80.

[20] R. M. Wojciechowski, A. Demenko, and J. K. Sykulski, "Inducted currents analysis in multiply connected conductors using reluctanceresistance networks," COMPEL, vol. 29, no. 4, pp. 908-918, Jul. 2010.

[21] A. Demenko, J. K. Sykulski, and R. Wojciechowski, "On the equivalence of finite element and finite integration formulations," IEEE Transactions on Magnetics, vol. 46, no. 8, pp. 3169-3172, 2010.

[22] A. Demenko and J. K. Sykulski, "Analogies between finite-difference and finite-element methods for scalar and vector potential formulations in magnetic field calculations," IEEE Transactions on Magnetics, vol. 52, no. 6, pp. 1-6, jun 2016

[23] A. Vishnevsky and A. Lapovok, "Boundary integral computation of electric fields in multielectrode galvanic systems using normally continuous elements," IEE Proceedings - Science, Measurement and Technology, vol. 147, no. 3, pp. 145-151, May 2000.

[24] A. Vishnevsky, A. Kalimov, and A. Lapovok, "Modeling magnetization using Whitney facet elements," IEEE Transactions on Magnetics, vol. 38, no. 2, pp. 489-492, Mar. 2002.

[25] A. Bossavit, "Whitney forms: a class of finite elements for threedimensional computations in electromagnetism," IEE Proceedings A Physical Science, Measurement and Instrumentation, Management and Education, Reviews, vol. 135, no. 8, p. 493, 1988

[26] V. Le-Van, "Développement de formulations intégrales de volume en magnétostatique," phdthesis, Université Grenoble Alpes, Grenoble, 2015.

[27] D. M. Araujo, "Plateforme de conception d'actionneurs: méthodes et outils pour le pré-dimensionnement d'actionneurs," phdthesis, Université de Grenoble, Apr. 2015.
[28] D. M. Araujo, G. Meunier, O. Chadebec, J. L. Coulomb, and L. Rondot, "3-D hybrid FEM \& BEM using whitney facet elements and independent loops," IEEE Transactions on Magnetics, vol. 51, no. 3, pp. 1-4, Mar. 2015.

[29] L. Urankar, "Vector potential and magnetic field of current-carying finite arc segment in analytical form, part II: Thin sheet approximation," IEEE Transactions on Magnetics, vol. 18, no. 3, pp. 911-917, May 1982.

[30] — " "Vector Potential and Magnetic Field od Current-Carrying Finite Arc Segment in Analytical Form, Part III: Exact Computation for Rectangular Cross Section," IEEE Transactions on Magnetics, vol. 18 , no. 6 , pp. 1860-1867, 1982.

[31] _ - "Vector potential and magnetic field of current-carrying finite arc segment in analytical form, part IV: General three-dimensional current density," IEEE Trans. Magn., vol. 20, no. 6, pp. 2145-2150, Nov. 1984

[32] _ - "Vector potential and magnetic field of current-carrying circular finite arc segment in analytical form - Part V. Polygon cross section," IEEE Transactions on Magnetics, vol. 26, no. 3, pp. 1171-1180, May 1990.

[33] H. Whitney, Geometric integration theory. Princeton: Princeton University Press, 1957

[34] L. O. Chua and P.-M. Lin, Computer-Aided Analysis of Electronic Circuits: Algorithms and Computational Techniques. New Jersey: Prentice-Hall, INC, 1975.

[35] S. Gumhold, S. Guthe, and W. Straßer., "Tetrahedral mesh compression with the cut-border machine," in Proceedings of the Visualization '99 Conference. IEEE Computer Society Press, Oct. 1999.

[36] T.-S. Nguyen, J.-M. Guichon, O. Chadebec, G. Meunier, and B. Vincent, "An independent loops search algorithm for solving inductive PEEC large problems," Progress In Electromagnetics Research M, vol. 23, no. November 2011, pp. 53-63, 2012.

[37] H. W. Derbas, J. M. Williams, A. C. Koenig, and S. D. Pekarek, "A comparison of nodal- and mesh-based magnetic equivalent circuit models," IEEE Transactions on Energy Conversion, vol. 24, no. 2, pp. 388-396, Jun. 2009.

[38] N. Takahashi, T. Nakata, K. Fujiwara, and T. Imai, "Investigation of effectiveness of edge elements," IEEE Trans. Magn., vol. 28, no. 2, pp. 1619-1622, Mar. 1992.

[39] C. Geuzaine and J.-F. Remacle, "Gmsh: A 3-D finite element mesh generator with built-in pre- and post-processing facilities," International Journal for Numerical Methods in Engineering, vol. 79, no. 11, pp. 1309-1331, Sep. 2009.

[40] P. Dular, C. Geuzaine, F. Henrotte, and W. Legros, "A general environment for the treatment of discrete problems and its application to the finite element method," IEEE Transactions on Magnetics, vol. 34, no. 5, pp. 3395-3398, Sep. 1998.

[41] E. Melgoza and R. Escarda, "Mapped infinite elements for the solution of open electric and magnetic field problems," in 2014 IEEE International Autumn Meeting on Power, Electronics and Computing (ROPEC). Institute of Electrical and Electronics Engineers (IEEE), Nov. 2014.

[42] A. S. Nunes, P. Kuo-peng, and M. G. V. Vanti, "Air Core Reactor Analysis Based on RNM Method," Proceedings of the Compumag 2013, 2013.

[43] A. S. Nunes, "Cálculo de perdas por fluxo disperso e de forças em transformadores e reatores através do método de rede de relutâncias," mathesis, Universidade Federal de Santa Catarina, 2013.

[44] B. P. S. Romain, "Modelisation des actionneurs electromagnetiques par reseaux de reluctances. creation d'un outil metier dedie au predimensionnement par optimisation," phdthesis, Université Joseph Fourier, 2006.

[45] H. C. Roters, Electromagnetic devices, 1st ed. Londion: John Wiley \& Sons, Inc., 1941. 